\title{
Interaction-aware risk assessment: focus on the lateral intention
}

\author{
Jorge Villagra, Antonio Artuñedo, Vinicius Trentin and Jorge Godoy
}

\begin{abstract}
To make the massive deployment of automated vehicles possible in complex urban environments, it is essential to provide them with the ability of making safe and useful decisions. To that end, it is necessary to improve their capability to infer the intentions of the surrounding vehicles and their associated collision risk for the ego-vehicle in complex driving scenes. This work shows the implementation and validation in simulation of a probabilistic approach to estimate the risk of driving under uncertain conditions, combining (i) intention estimations and (ii) the expected behaviour of vehicles according to the topology and the subsequent traffic rules of the considered driving scenario. Promising results in terms of success rate and prediction horizon have been obtained testing the proposed approach in driving situations where lateral intention estimation is relevant, namely in multi-lane roundabouts and highways.
\end{abstract}

\section{INTRODUCTION}

In typical urban driving scenes such as intersections or roundabouts, a large number of traffic agents can present a collision risk for an automated vehicle (AV). It may be extremely difficult to predict individually and accurately the trajectories of each agent in scenes involving a large number of them, in which their behaviours have clear interactions.

The most popular paradigm for estimating collision risk in robotics, and therefor in the AV community, is based on the idea of "motion prediction + collision detection", composed of two steps: (i) individually predict possible future trajectories for all moving entities at the scene, (ii) detect collisions between pairs of trajectories, and derive a risk estimate based on the total possibility of collision. However, the interplay among different types of moving entities cannot be neglected in many urban environments. In this context, interaction-aware risk assessment is a promising research field, where different modelling and risk inference strategies have been proposed. Among them, the use of Dynamic Bayesian Networks (DBN) allows to efficiently model the interconnected behaviour of vehicles, while taking into consideration system and measurement uncertainties.

The work of [11] is a theoretically scalable and generic DBN approach that can explicitly consider any driving context and its associated traffic rules. However, it has only been tested for longitudinal risk assessment and with pairs of vehicles.

Following this approach, the present work incorporates a model for the lateral intention of vehicles within the existing network. The easy-to-compute utility-based model [1] is introduced and can be used in driving scenarios where

Jorge Villagra, Antonio Artuñedo, Vinicius Trentin and Jorge Godoy are with the Autopia Program, Centre for Automation and Robotics (CSICUPM), Ctra. Campo Real, km 0.200, Arganda del Rey (Madrid), Spain. both longitudinal and lateral dynamics may influence risk assessment. To showcase its potential, the paper assesses the strategy performance in different complex driving situations, such as highways and multi-lane roundabouts, where at least 3 vehicles interact.

The outline of the paper is as follows. A summary of the most relevant related works are presented in Section II. Section III describes how the behaviour of vehicles interactions are modelled using a DBN. Section IV is specifically devoted to the lane change modelling and its implication in the lateral expectation and intention estimation. To infer the outputs of the DBN, a particle filter is implemented, for which some basic guidelines are provided in Section V. The applicability of the proposed approach is validated in different situations where lateral interactions are relevant, such as highways or roundabouts.

\section{RELATED WORK}

Motion prediction techniques, according to [12], can be classified in 3 basic categories: (i) physical models, where vehicles motion is estimated using the laws of physics, without considering the existence of other vehicles, being the main differences among them their uncertainty management -single path simulation [14], Gaussian noise simulation [18] or Monte Carlo simulations [2]-; (ii) maneuvers-based models (e.g. [3][19][20]), where vehicles motion depends on the maneuvers that their drivers intend to perform, using for that physical variables -speed, position, direction, road information -geometry, topology, speed limits, traffic laws- or driver behavior -head movement, driving style; (iii) interaction-aware models, based on the principle that to perform a certain maneuver, vehicles recognize their mutual presence, either with computation-intensive approaches -e.g. using hidden and coupled Markov[4], or finding solutions to specific simplified driving situations -e.g. overtaking [24].

Physical models have two main drawbacks: the motion prediction they provide are too short term, and, as a result of this, they are not able to foresee any change in the car motion caused by the execution of a particular maneuver or by changes due to external factors. Although maneuver-based models can predict the intention of a driver by adjusting the trajectories and states, they do not take into account the dependencies between vehicles. As a result, interactionaware seem to be the most appropriate approach for driving situations with a high number of interacting agents.

Several recent papers have proposed machine learning techniques to estimate local spatio-temporal interactions among vehicles as an element of the decision-making architecture in an automated vehicle (e.g. [22], [13]). However, 
exhaustive and well prepared datasets are required to achieve good results, which, in addition, are often used in end-to-end solutions, where driving risk assessment is bypassed [10]. The work of [11] is an original proposal for this kind of applications, since it allows solving the risk inference taking into account the uncertainty, the topology and the context of the situation according to traffic regulations. However, the validation of this promising approach was done only taking into account 2 vehicles and in a very specific case: unsignalized T-shaped intersections. The algorithm, being in principle effective and scalable, needed to be enlarged for driving situations where lateral intention estimation is key.

Just as longitudinal intention is modelled considering simple interacting principles, a lane-change model is required to cope with the vehicles interactions in a cost-efficient manner. The literature for microscopic simulation [5][17] has been fed in the last 30 years with different approaches to efficiently simulate the driver lateral behaviour in presence of traffic. Thus, lane-changing models can be categorized into four groups [21]: rule-based models [6], [7], [9], discretechoice-based models [1], [25], artificial intelligence models [28], [23], and incentive-based models [8]. Incentive-based strategies (whose most relevant representative is the MOBIL model) have the ability to introduce some driver variability, but is is unclear its behaviour in busy scenarios, as it only represents operational lane-changing decisions and do not describe strategical and tactical aspects. As already mentioned, AI-enabled models require exhaustive data for their deployment, which in addition are sometimes untraceable. Rule-based and discrete-choice-based models appear to be the most popular approaches, due to their simplicity. Although rule-based models have a small number of parameters to tune, their outputs are expressed as binary variables, which do not fit with the underlying probabilistic framework of [11]. As a result, one of the better known discrete-choicebased models [1] has been implemented and integrated to infer lateral intentions and the resulting risk assessment of lane-changing driving situations.

\section{DYNAMIC BAYESIAN NETWORK}

The Dynamic Bayesian Network model used is inspired from [11] and considers four state variable types: (i) expected manoeuvre $\left(E_{t}^{n}\right)$, (ii) intended manoeuvre $\left(I_{t}^{n}\right)$, (iii) physical state $\left(\Phi_{t}^{n}\right)$ and (iv) measurements $\left(Z_{t}^{n}\right)$, where the expected and intentional manoeuvres are unknown and intangible variables, unlike the physical state. The generalized distribution defining the the network in a time interval $0: T$ can be expressed as follows:

$$
\begin{aligned}
& P\left(\mathbf{E}_{\mathbf{0}: \mathbf{T}}, \mathbf{I}_{0: \mathbf{T}}, \Phi_{0: \mathbf{T}}, \mathbf{Z}_{0: \mathbf{T}}\right)=P\left(\mathbf{E}_{\mathbf{0}}, \mathbf{I}_{0}, \Phi_{0}, \mathbf{Z}_{\mathbf{0}}\right) \times \\
& \times \prod_{t=1}^{T} \times \prod_{n=1}^{N}\left[P\left(E_{t}^{n} \mid \mathbf{I}_{\mathbf{t}-1} \Phi_{\mathbf{t}-\mathbf{1}}\right) \times P\left(I_{t}^{n} \mid \Phi_{t-1}^{n} I_{t-1}^{n} E_{1}^{n}\right) \times\right. \\
& \left.\times P\left(\Phi_{t}^{n} \mid \Phi_{t-1}^{n} I_{t-1}^{n} I_{t}^{n}\right) \times P\left(Z_{t}^{n} \mid \Phi_{t}^{n}\right)\right]
\end{aligned}
$$

In real driving scenarios the behaviour of each vehicle is conditioned by the behaviour of the rest vehicles. As a result, the network is instantiated for each of the $n$ vehicles, being all these instantiations interrelated. The bold variables reflect the dependencies of vehicle $n$ with respect to the other vehicles

The expected manoeuvre represents the expected behaviour of every vehicle according to traffic rules. It is a generic tool to introduce the geometrical and topological characteristics of the driving scene and the associated conventions for safe vehicles interrelation. This variable is therefore different when facing a roundabout, driving in a highway, or approaching traffic lights, and it is divided into two types: longitudinal and lateral. The former model the probability that the vehicle should stop at some point. The latter, which is the focus of this paper, interprets when a vehicle is expected to make a lane change without hindering traffic.

The intended manoeuvre has a certain similarity to the expected manoeuvre since it models the same behaviour from the perspective of the driver himself. However, the main difference lie in considering the current and previous motion trend of the vehicle, rather than the most likely evolution given a certain traffic scenario. In other words, expectation express what it should normally happens, and intention, what it seems to be happening (or about to happen). As in the case of expected manoeuvre, it is divided into longitudinal and lateral and models the probability to keep going or stop, in the first case, and remain or change lane, in the second.

Note that the physical state (pose and velocity) of every involved vehicle is inferred using certain modelling hypothesis from the intention estimation [11], [26]. Unlike these probabilistically estimated state variables, pose and velocity measurements are directly extracted from the sensors and compared with the estimated physical state. Both types of variables are used in a particle filter (see more details in Section V) to balance observation and prediction according to their associated uncertainty. As a result, if measurements and physical state coincide to a lesser or greater degree, a lesser or greater probability can be assumed that the estimates of expectation and intention are valid.

\section{LATERAL EXPECTATION AND INTENTION}

The computation details of most of the aforementioned variables are sufficiently covered in [11] and therefore not included here. However, the lane change model has been introduced in this work, and therefore the procedure to estimate lateral expectation and intention is described below:

1) The closer vehicle ahead the ego-vehicle, as well as the leader and rear vehicles in the adjacent left and right are identified.

2) The deceleration caused by the leader vehicle of the current lane is computed using the expression $a=\frac{\alpha v \Delta v}{\Delta x^{2}}$, where $v$ is the speed of the vehicle $n, \Delta v$ is the difference between the speed of $n$ and that of the leader, $\Delta x$ is the gap between both vehicles and $\alpha$ is a design coefficient [16].

3) Following the value of $a$ and the context, the most likely lane to be followed is selected as follows: 
a) if $a<0$, the ego-vehicle is potentially forced to change of lane. The probability to turn right or left lane is determined by $P_{c}(j)=\frac{e^{a_{j}}}{\sum_{i=1, r}^{N} e^{a_{i}}}$, where $i$ and $j$ can be left or right, and $a_{j}$ is computed as for the ego-vehicle deceleration, using the values of the leading vehicle of the corresponding lane.

b) If $a<0$ and $n$ is not in the right lane, the one on the right is chosen as the new lane, since, if possible, either by traffic regulations on a highway or by the intention of leaving a roundabout, it is likely that the driver wishes to make a change in that direction.

c) If $a<0$ and $n$ is in the right lane, 'STAY' is chosen as the new expectation value, finishing the calculation at this point.

4) Once the most likely lane has been chosen, it is necessary to ensure that the change is feasible. To that end, the gap acceptance probability $P_{a}$ is computed for both the leader and rear vehicles using $P_{a}(l, r)=$ $1-e^{-\lambda\left(t_{l, r}-T\right)}$, where indexes $l, r$ refer to leader and rear, respectively, $t_{l, r}$ are the actual time gaps between the ego-vehicle and the leader/rear vehicles, $T$ is a socalled critical time gap, and $\lambda$ is a design coefficient [16]. The probability that the total gap is sufficient to change the lane is computed using the product of the two probabilities $P_{a}(l)$ and $P_{a}(r)$.

5) The final expectation of changing lane $E c_{t}^{n}$ is obtained from the comparison of a random variable and the probability computed in the previous point.

Following the idea presented in [11], the behavior table I is used to estimate the intended lateral maneuver, taking as inputs the values of the current expectation and intention of the previous moment. Note that the expected maneuvre $E c_{t}^{n}$ takes one of the following 2 values:

- STAY: The vehicle should not make a lane change because traffic conditions do not allow it. This may be due to the rest of the lanes being congested, there is not enough space (gap) between adjacent vehicles, the current lane is the most suitable for the speed that the vehicle is carrying, etc..

- CHANGE: The vehicle can make a lane change without hindering traffic or causing an accident. In this case there are two possible sub-states, which would be the possibility to change to the right or left.

TABLE I: Lateral Intention behaviour table

\begin{tabular}{|c|c|c|}
\hline $\mathbf{I c}_{\mathbf{t}-\mathbf{1}}^{\mathbf{n}}$ & $\mathbf{E} \mathbf{c}_{\mathbf{t}}^{\mathbf{n}}$ & $\mathbf{P}\left(\left[\mathbf{I} \mathbf{c}_{\mathbf{t}}^{\mathbf{n}}=\mathbf{c h a n g e}\right] \mid \mathbf{I c}_{\mathbf{t}-\mathbf{1}}^{\mathbf{n}} \mathbf{E c}_{\mathbf{t}}^{\mathbf{n}}\right)$ \\
\hline$S T A Y$ & STAY & 0.1 \\
\hline STAY & CHANGE & 0.2 \\
\hline CHANGE & STAY & 0.5 \\
\hline CHANGE & CHANGE & 0.9 \\
\hline
\end{tabular}

The values shown in the table are an approximation to the behavior that could be expected and have been adjusted experimentally.

\section{PARTicle Filter}

As the exact inference of expression (1) is not tractable, a Montecarlo-based approximate inference method has been used. For the detection of risk situations, the manoeuvre expected to be executed by the driver is compared with his/her intentions in a given context (e.g. highway, roundabouts, etc). Each of the possible states of the system is contained in a particle, which has an associated weight dependent on its proximity to reality.

Each of the $N$ particles gives a random value to the hidden variables, difficult to infer directly from externally observable variables. In our case, the hidden variables are the intention and expectation of each vehicle. From them, a prediction of the observable variables is conducted. Once this is done for all the particles, the next step is to give a weight to each particle -update. The weight given to each particle is greater or lesser if its prediction is more or less accurate, respectively. Thus, it is a measure of the acceptance of the hidden variables that are estimated by that particle. Weights can end up being very disparate, as they evolve over the initial weight. An Importance Resampling procedure is used to identify the useless or erratic particles (with negligible weight) and replace them efficiently, thus mitigating sample degeneracy (and potentially sample deprivation), while avoiding impoverishment. Finally, to compute the resulting intentions and expectations, the weights of all the particles whose intention/expectation values are CHANGE (in case of lateral estimation) are selected and normalized with respect to the overall number of particles.

This procedure ends with the final prediction of the hidden variables for each of the vehicles. This risk is thereafter obtained by taking the weights of the particles whose expectation and intention differ. This occurs, for example, if the intention is to stay in the lane in a roundabout and the expectation is to change, since this implies that, under current traffic conditions and rules, the vehicle should remain in the lane and yet intends to occupy another lane. The result of this calculation is the indicator of collision risk, which is considered relevant from a certain threshold $\varepsilon_{r}$ [11], [26].

\section{Simulation RESUlts}

To assess the performance of the proposed risk estimation strategy, two different scenarios where lateral expectation is relevant have been considered: (i) a highway and (b) a multilane roundabout. The methodology followed to evaluate the proposed risk assessment algorithm has followed the following 2 main steps:

1) A series of driving situations are conducted for each scenario, where both collisions and non-colliding situations are recreated, collecting logs with the positions and speeds of ever vehicle over time. SUMO open source simulator [15] and TraCI interface [27] to Matlab are used to that end.

2) The algorithm is run with the recorded data to produce the evolution of intention, expectation and risk values over time. The parameters used are collected in Table 
II. This step is performed several times for each simulation, since the algorithm is based on a stochastic approach and the consistency of its behaviour needs to be checked in different sets of simulations.

\begin{tabular}{|l|c|}
\hline Parameter name & Value \\
\hline Deceleration param. for lateral gap acceptance $(\alpha)$ & 25 \\
\hline Critical time gap $(\mathrm{T})$ & 0.7 \\
\hline Time constant in gap acceptance probabilit $(\lambda)$ & 0.78 \\
\hline Risk threshold $\left(\varepsilon_{r}\right)$ & 0.3 \\
\hline
\end{tabular}

TABLE II: Design parameters

Once the results are obtained, they are classified by successes (the algorithm has correctly detected whether vehicles generate a hazard) or false positives (risk is detected in safe situations).

\section{A. Highway}

With the highway scenario it is intended to test the risk estimation algorithm in environments where the lane change of a vehicle could cause a hazardous situation. The conducted tests focus on three main cases, shown in FIg. 1.

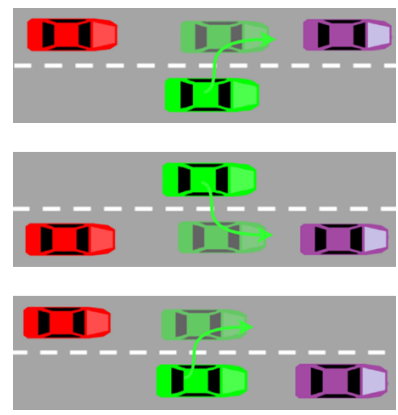

Fig. 1: Three considered cases in the highway scenario

A particular vehicle (ego-vehicle) is asked to change its lane without taking into account traffic conditions, thus generating dangerous situations. The rest of vehicles perform the overtaking manoeuvres simulating the human behaviour. These vehicles move at higher speeds than the vehicle that executes the manoeuvre in an unsafe way, so that, when changing lanes, the ego-vehicle generates danger in the vehicles that circulate at a higher speed. To illustrate these movements in the simulations, Fig. 2 presents one of these cases step by step from top to bottom. As can be seen, this scenario consists of a one-way road with three parallel lanes.

Each scenario for each situation (either danger situation for the main vehicle or not) is simulated five times obtaining a total amount of 30 tests $(3$ environments $\times 2$ situations $\times 5$ simulations). In this case, the risk situations have been correctly detected by the algorithm in the 30 tests: in 15 of them the risky situation have been detected and in the other 15 tests, no dangerous situation has been noticed.

Regarding the risk prediction time, all risky situations have been detected between 0.9 and $1.1 \mathrm{~s}$ before the lane change ends, thus providing valuable information for the decision system to be able to react before a possible collision. Note that the simulated lane changes last $1.5 \mathrm{~s}$.

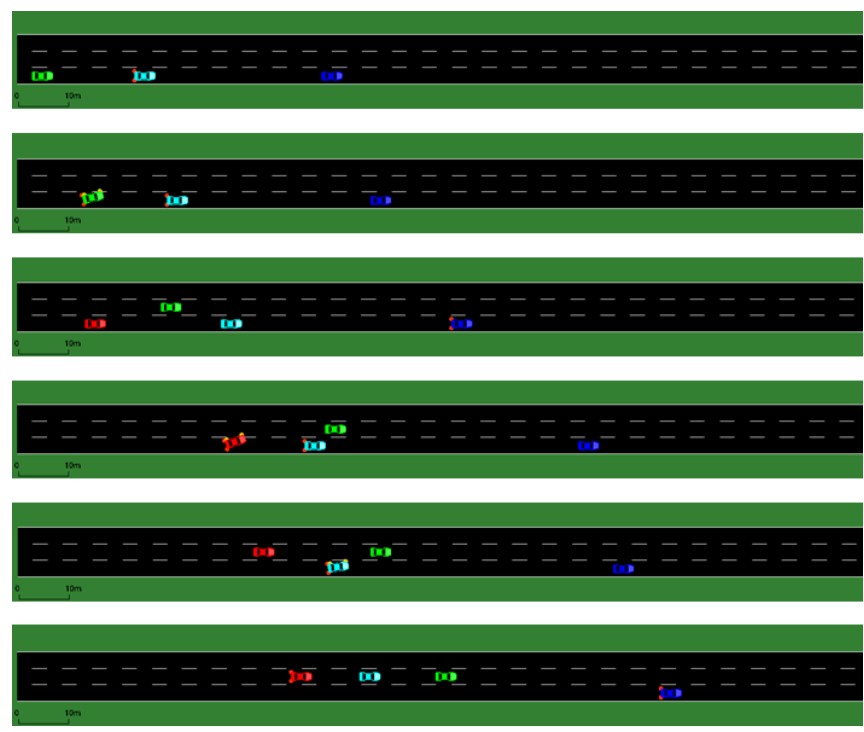

Fig. 2: Highway scenario

Figure 3 shows one example of the resulting lateral expectation, intention and risk estimation in one of the scenarios represented in figure 2. Note that the risk indicator starts increasing when the intention of the blue vehicle also raises, as the lateral expectation is that this vehicle stays in the original lane. In this particular case the algorithm is able to detect the hazardous situation $1.05 \mathrm{~s}$ in advance to a possible collision.

\section{B. Roundabout}

The roundabout scenario includes three lanes and the manoeuvres to be performed by the vehicles are the same than those of the highway scenario (see figure 4). However, a new complexity with respect to the previous scenario is introduced: the angular speed of the vehicles make difficult to differentiate whether a vehicle is manoeuvring a lane change or not, which may eventually cause the indicator to have generally larger values.

The design of the roundabout scenario in SUMO entails some limitations of the realism of the simulated environment. As can be seen in Fig. 4, roundabouts in SUMO are not modelled with a curvilinear path, but as a concatenation of straight segments. This representation limitation makes it difficult to model the position of the vehicle, since it contains completely straight sections and areas in which the turn is quite abrupt. After adjusting the modelling of the vehicle motion as much as possible to reality, risk peaks continue to occur at points where the direction of the vehicle changes, due to inability of the algorithm to predict these abrupt changes.

Despite of this supplementary complexity, the risk estimation algorithm is able to detect risky situations properly in all the proposed hazardous situations, as shown in table III. Nevertheless, 3 of the 15 cases in safe situations are detected as risky. As aforementioned, this false positive cases can be caused by the way in which roundabouts are modelled in SUMO. 


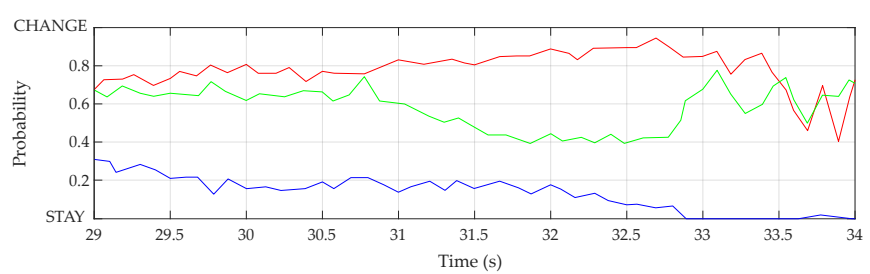

(a) Lateral expectation

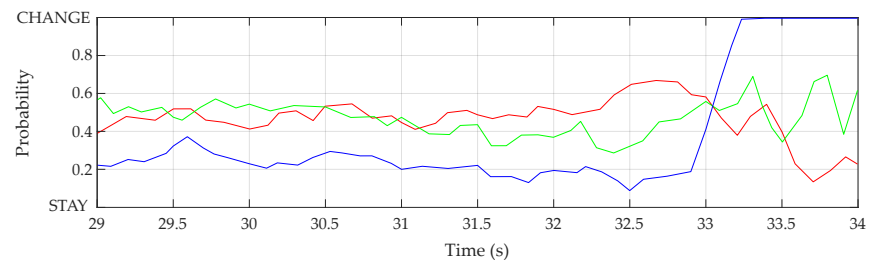

(b) Lateral intention

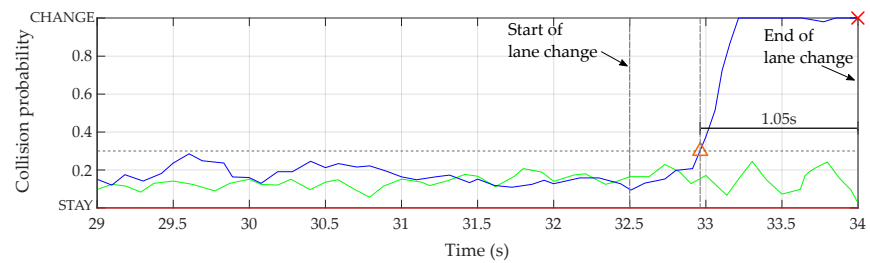

(c) Lateral risk indicator

Fig. 3: Results of the lateral risk indicator in the highway scenario

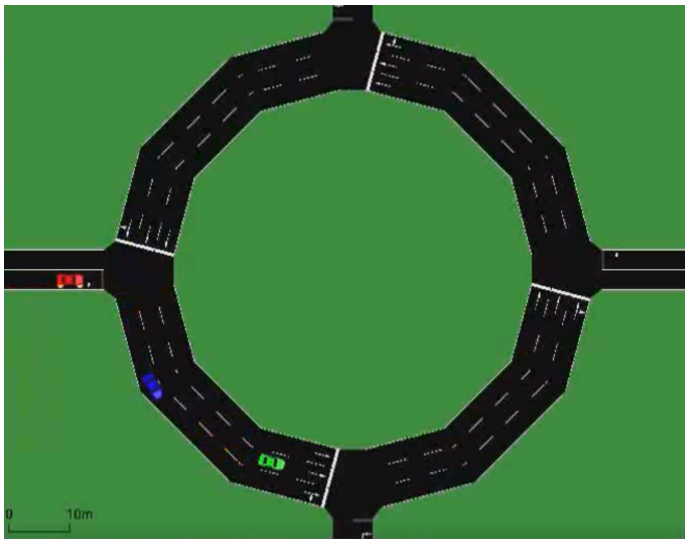

(a) roundabout scenario at $\mathrm{t}=31 \mathrm{~s}$

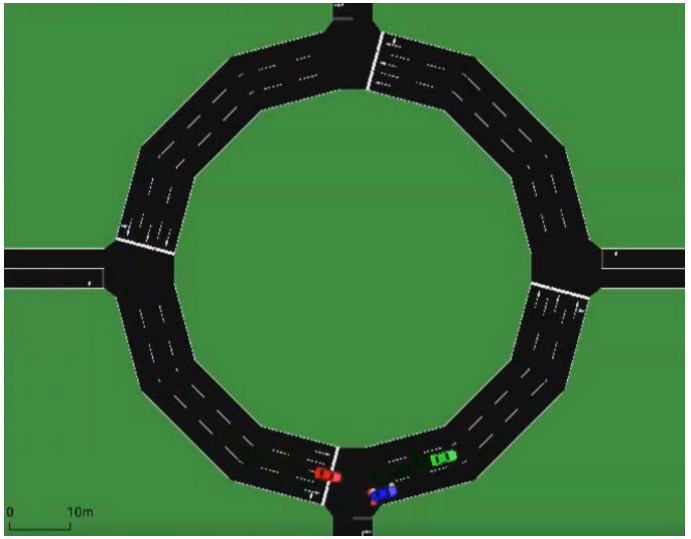

(b) roundabout scenario at $\mathrm{t}=38 \mathrm{~s}$

Fig. 4: Roundabout scenario sequence

\begin{tabular}{|c|c|c|c|}
\hline Situation & Risk detected & Risk no detected & Success rate (\%) \\
\hline Hazardous & 15 & 0 & 100 \\
\hline Safe & 3 & 12 & 80 \\
\hline
\end{tabular}

TABLE III: Roundabout results

Note that the simulated vehicles perform the lane change manoeuvre in $1.5 \mathrm{~s}$, as in the highway scenario. The reaction times in the conducted simulations for the roundabout scenario are between 0.8 and $1.2 \mathrm{~s}$.

The results of one of the simulations performed in this scenario is shown in figure 5 . In this case, it can be noticed how the risk indicator exceeds the threshold risk value $1.13 \mathrm{~s}$ before the possible collision.

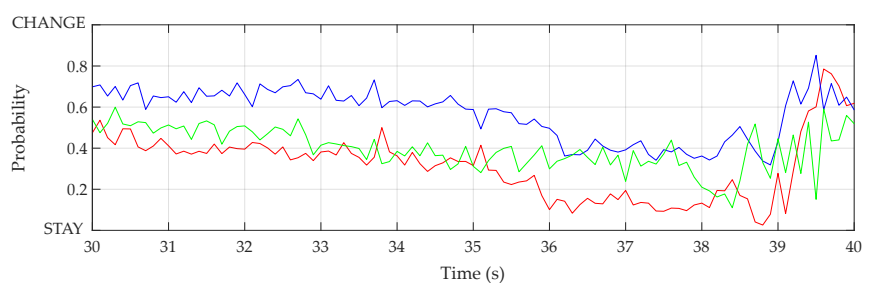

(a) Lateral expectation

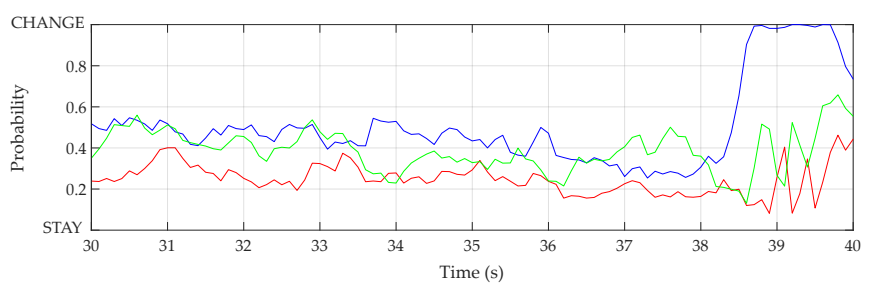

(b) Lateral intention

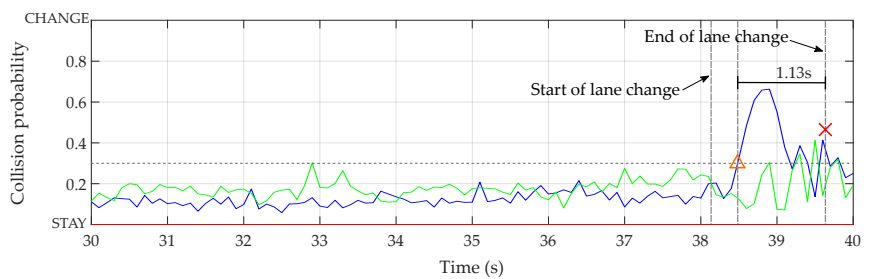

(c) Lateral risk indicator

Fig. 5: Results of the lateral risk indicator in the highway scenario

Table IV summarizes the data obtained in the 2 considered driving scenarios, including detection success rate and different metrics about the time in advance the risk is identified. Note that although the roundabout scenario entails an additional degree of complexity, the mean prediction times are very similar to the ones obtained in the highway.

\begin{tabular}{|c|c|l|l|l|l|}
\cline { 3 - 6 } \multicolumn{1}{c|}{} & \multirow{2}{*}{ Success rate } & \multicolumn{4}{|c|}{ Prediction time } \\
\cline { 3 - 6 } \multicolumn{1}{c|}{} & & $\min$ & $\max$ & mean & std \\
\hline Highway & $100 \%$ & 0.9 & 1.1 & 1.03 & 0.04 \\
\hline Roundabout & $90 \%$ & 0.8 & 1.2 & 1.02 & 0.09 \\
\hline
\end{tabular}

TABLE IV: Summary of results 


\section{CONCLUding REMARKS}

The results obtained show the feasibility of the proposed method to assess risk of collision considering lateral intention. The implemented particle filter was able to predict the risk situation in $95 \%$ of the tested driving environments with acceptable margins of time.

As it is expected to detect possible collisions at about $1.5 \mathrm{~s}$ in advance, further studies will be considered in two main directions: (i) the number of particles becomes a key ingredient to enhance the risk detection times, and therefore a more efficient implementation of the particle filter and its coupling with motion prediction techniques will be investigated; (ii) the use of real-word data experiments will be used to fine-tune model parameters and to test its behaviour on a more realistic simulation environment (like ScanerStudio).

\section{ACKNOWLEDGMENT}

This work has been partially funded by the Spanish Ministry of Science, Innovation and Universities with National Project COGDRIVE (DPI2017-86915-C3-1-R), the Community of Madrid through SEGVAUTO 4.0-CM (S2018EMT-4362) Programme, and by the European Commission through the Projects NEWCONTROL (ECSEL-826653-2) and SECREDAS (ECSEL-783119-2).

\section{REFERENCES}

[1] K. I. Ahmed. Modeling drivers' acceleration and lane changing behavior. PhD thesis, Massachusetts Institute of Technology, 1999.

[2] M. Althoff and A. Mergel. Comparison of markov chain abstraction and monte carlo simulation for the safety assessment of autonomous cars. IEEE Transactions on Intelligent Transportation Systems, 12(4):1237-1247, 2011.

[3] G. S. Aoude et al. Driver behavior classification at intersections and validation on large naturalistic data set. IEEE Transactions on Intelligent Transportation Systems, 13(2):724-736, 2012.

[4] M. Brand, N. Oliver, and A. Pentland. Coupled hidden markov models for complex action recognition. In Proc. Comp. Vision and Pattern Recognition, pages 994-999, 1997.

[5] J. Erdmann. Sumo's lane-changing model. In Modeling Mobility with Open Data, pages 105-123. Springer, 2015.

[6] P. G. Gipps. A model for the structure of lane-changing decisions. Transportation Research Part B: Methodological, 20(5):403414, 1986.

[7] P. Hidas. Modelling vehicle interactions in microscopic simulation of merging and weaving. Transportation Research Part C: Emerging Technologies, 13(1):37-62, 2005.

[8] A. Kesting, M. Treiber, and D. Helbing. General lane-changing model mobil for car-following models. Transportation Research Record, 1999(1):86-94, 2007.

[9] H. Kita. A merging-giveway interaction model of cars in a merging section: a game theoretic analysis. Transportation Research Part A: Policy and Practice, 33(3-4):305-312, 1999.

[10] A. Kuefler, J. Morton, T. Wheeler, and M. Kochenderfer. Imitating driver behavior with generative adversarial networks. In 2017 IEEE Intelligent Vehicles Symposium (IV), pages 204-211. IEEE, 2017.

[11] S. Lefèvre, C. Laugier, and J. Ibañez-Guzmán. Risk assessment at road intersections: Comparing intention and expectation. In IEEE Intelligent Vehicles Symposium (IV), pages 165-171, 2012.

[12] S. Lefèvre, D. Vasquez, and C. Laugier. A survey on motion prediction and risk assessment for intelligent vehicles. Robomech Journal, 1(1):1, 2014.

[13] J. Li, H. Ma, and M. Tomizuka. Interaction-aware multi-agent tracking and probabilistic behavior prediction via adversarial learning. In 2019 International Conference on Robotics and Automation (ICRA), pages 6658-6664. IEEE, 2019.
[14] C.-F. Lin et al. Vehicle dynamics and external disturbance estimation for vehicle path prediction. IEEE Transactions on Control Systems Technology, 8(3):508-518, 2000.

[15] P. A. Lopez, M. Behrisch, L. Bieker-Walz, J. Erdmann, Y.-P. Flötteröd, R. Hilbrich, L. Lücken, J. Rummel, P. Wagner, and E. WieBner. Microscopic traffic simulation using sumo. In 2018 21st International Conference on Intelligent Transportation Systems (ITSC), pages 25752582. IEEE, 2018.

[16] T. V. Mathew. Lane changing models. Transportation systems engineering, pages 15-1, 2014.

[17] S. Moridpour, M. Sarvi, and G. Rose. Lane changing models: a critical review. Transportation letters, 2(3):157-173, 2010.

[18] K. P. Murphy. Dynamic bayesian networks: representation, inference and learning. $\mathrm{PhD}$ thesis, University of California, Berkeley, 2002.

[19] M. G. Ortiz et al. Behavior prediction at multiple time-scales in innercity scenarios. In IEEE Intelligent Vehicles Symposium (IV), pages 1068-1073, 2011.

[20] D. J. Phillips, T. A. Wheeler, and M. J. Kochenderfer. Generalizable intention prediction of human drivers at intersections. In 2017 IEEE Intelligent Vehicles Symposium (IV), pages 1665-1670. IEEE, 2017.

[21] M. Rahman, M. Chowdhury, Y. Xie, and Y. He. Review of microscopic lane-changing models and future research opportunities. IEEE transactions on intelligent transportation systems, 14(4):1942-1956, 2013.

[22] W. Schwarting, J. Alonso-Mora, and D. Rus. Planning and decisionmaking for autonomous vehicles. Annual Review of Control, Robotics, and Autonomous Systems, 2018.

[23] J. Tang, F. Liu, W. Zhang, R. Ke, and Y. Zou. Lane-changes prediction based on adaptive fuzzy neural network. Expert Systems with Applications, 91:452-463, 2018.

[24] C. Tay. Analysis of dynamic scenes: application to driving assistance. L'Institut Poly technique de Grenoble, FranceDèpt. Telecommun. 2009

[25] T. Toledo, H. N. Koutsopoulos, and M. E. Ben-Akiva. Modeling integrated lane-changing behavior. Transportation Research Record, 1857(1):30-38, 2003

[26] J. Villagra, M. Perarnau, J. Godoy, and A. Artunedo. Validación de una estrategia para la estimación del riesgo en intersecciones con vehículos conectados. Actas de las XXXIX Jornadas de Automática, Badajoz 5-7 de Septiembre de 2018, 2018.

[27] A. Wegener, M. Piórkowski, M. Raya, H. Hellbrück, S. Fischer, and J.-P. Hubaux. Traci: an interface for coupling road traffic and network simulators. In Proceedings of the 11th communications and networking simulation symposium, pages 155-163, 2008.

[28] J. Wu, M. Brackstone, and M. McDonald. Fuzzy sets and systems for a motorway microscopic simulation model. Fuzzy sets and systems, 116(1):65-76, 2000. 